Revista Española de Antropología Americana ISSN: 0556-6533

https://doi.org/10.5209/REAA.61980

\title{
Historia y arqueología de la formación del altepetl en la Cuenca de México durante el Posclásico Medio
}

\author{
Raúl García Chávez y Natalia Moragas Segura²
}

Recibido: 10 de mayo de 2017 / Aceptado: 25 de julio de 2017

Resumen. En este trabajo se considera el proceso de desarrollo sociopolítico de los altepeme de la Cuenca de México durante el Posclásico Medio. Las sociedades chichimecas resultan especialmente interesantes para comprender los diversos procesos de desarrollo sociocultural en un espacio y tiempo bien definidos. El estudio de los diversos altepeme nos permitirá comprender las diferentes dinámicas sociopolíticas que se van a desarrollar por distintos grupos en aras de consolidarse en el poder.

Palabras clave: Cuenca de México, altepetl, Posclásico Medio.

\section{[en] History and Archaeology of the Formation of the Altepetl in the Basin} of Mexico during the Middle Postclassic Period

\begin{abstract}
In this paper, we consider the socio-political development process of the altepetl in the Mexican Basin during the Middle Postclassic Period. Chichimec societies are especially interesting to understand the various processes of socio-cultural development in a well-defined space and time. The study of the various altepeme will allow us to understand the different socio-political dynamics used by different groups in order to consolidate themselves in power.
\end{abstract}

Palabras clave: Basin of Mexico, altepetl, Middle Postclassic.

Sumario. 1. Introducción. 2. El desarrollo del altepetl durante el Posclásico Temprano (1150-1325). 3. La caracterización de las sociedades chichimecas desde la jefatura/cacicazgo hasta el Estado. 4. Conclusiones. 5. Referencias.

\section{Introducción}

La Cuenca de México es un área significativa para la arqueología mundial por sus características medioambientales específicas y por haber conjuntado no sólo una larga ocupación humana sino también elevadas cantidades de población. Su trayectoria en el tiempo ejemplifica claramente el proceso de una creciente complejidad social, permitiendo estudiar numerosos aspectos de desarrollo sociocultural que son de interés de la arqueología, como la sedentarización, la constitución de los centros urbanos y su expansión territorial, así como el colapso de los mismos; la Cuenca de México

\footnotetext{
1 Centro INAH Estado de México. ernesto_garcia@inah.gob.mx

2 Depto. de Historia y Arqueología, Universitat de Barcelona. nataliamoragas@ub.edu
} 
constituye un espacio privilegiado para comprender los procesos sociales y culturales de grandes grupos a lo largo del tiempo (Sanders et al. 1979). Siendo el lugar en donde se originaron y desarrollaron importantes sociedades estatales, es normal que gran parte de las investigaciones arqueológicas se hayan centrado en el estudio de las grandes ciudades de Teotihuacan y México-Tenochtitlan, sin olvidar otros centros como Cuicuilco o Azcapotzalco. Tales investigaciones se centran en momentos históricos en los que toda la Cuenca de México se encuentra bajo el dominio ideológico y económico de dichos centros y en el que la cultura material se muestra mucho más homogénea, evidenciando su poder e influencia sobre el territorio y el modo en que ejercen el control de las rutas comerciales y de la distribución de los productos.

En el estudio de las sociedades del Posclásico en la Cuenca de México se puede inferir una evolución política y social continuada desde formas más simples hasta las más complejas. En trabajos anteriores, se ha conceptualizado la evolución sociopolítica de la Cuenca de México como fases que alternaban periodos de homogeneización y momentos de heterogeneidad cultural, sobre todo en lo que se refiere a la esfera política, creando un buen punto para el inicio del análisis a nivel macroespacial (Charlton y Nichols 1997 a y b). Mientras que los estudios sobre Teotihuacan o Tenochtitlan no discuten el hecho de encontrarnos con sociedades estatales consolidadas, con un firme predominio en el territorio circundante y una política de expansión de su modelo, el estudio de los periodos intermedios es mucho más complejo, ya que nos movemos en situaciones con registros arqueológicos menos visibles y con fronteras más difusas (Moragas 2005) ${ }^{3}$.

Sin embargo, y dentro de este contexto general, el estudio de las sociedades chichimecas no ha recibido el mismo interés; dicho de otra manera: el acercamiento a su problemática específica es uno de los temas pendientes de la arqueología de la Cuenca de México. Para un acercamiento inicial desde las fuentes históricas, se pueden referir algunos trabajos ya clásicos como los de Carrasco (1984, 1996); Carrasco y Broda (1976, 1978); Caso (1967); Castillo (1984); Corona (1973); Davies (1973, 1980, 1987); Gibson (1981); Hodge (1984); Jiménez Moreno (1961) Kirchhoff (1954-55, 1985); Kirchhoff et al. (1976); Reyes García (1988); Pérez Rocha (1982); Smith (1996); Zantwijk (1969, 1975, 1985). Así mismo existen algunas tipologías cerámicas, si bien no abarcan toda la complejidad del período (Brumfiel 1976; Franco 1945; Gamio et al. 1990 [1921]; Griffin y Espejo 1947; Hodge y Minc 1990; Noguera 1935; O’Neill 1962; Parsons 1966; Sanders et al. 1979; Sejourné 1970; Smith y Hodge 1994). Pero cabe recordar que en el uso de las fuentes históricas no podemos obviar que hay que confrontar «los mitos de alteridad» presentes en la concepción propia de la interpretación de lo chichimeca (barbarie) frente lo urbano (civilizado) en los textos de cronistas hispanos y mestizos en los siglos XVI y XVII, influyendo en nuestro conocimiento e interpretación de las culturas indígenas americanas (Pérez Flores 2013; Santamarina 2015).

Siguiendo las líneas de trabajo de Charlton y Nichols (1997b: 198), analizar la evolución de las sociedades de la Cuenca de México como formaciones estatales

\footnotetext{
3 Este ha sido uno de los puntos de discusión clave en la definición de la evolución sociopolítica de la cuenca de México y de debate entre los autores de este texto en lo que se refiere a la visión histórica de los procesos arqueológicos vistos desde Teotihuacan y el sur de la Cuenca de México. Revisar las presencias y ausencias de determinados materiales y los cambios en el patrón de asentamiento reflejan dinámicas políticas distintas dependiendo del impacto, en el caso que discutimos, del colapso teotihuacano y la reestructuración del territorio bajo nuevos liderazgos.
} 
centralizadas de este periodo resulta todo un reto. Las sociedades chichimecas constituyen un caso de estudio muy interesante por su incorporación a un territorio que había sufrido variados e intensos fenómenos de concentración y disgregación sociopolítica y con un corpus cultural e ideológico bien consolidado a lo largo de más de mil años. En el caso del Posclásico Temprano en la Cuenca de México, las unidades políticas tienen algunas de las características que se suponen en un Estado, pero con un tamaño mucho menor que las sociedades que las anteceden y preceden ${ }^{4}$.

La propia definición de qué es y no es un altepetl ha producido una copiosa bibliografía, abordando desde la etimología del término y la descripción de sus características hasta su transformación a lo largo de los siglos. Las investigaciones han puesto énfasis en los aspectos urbanos del concepto, siendo más fácil de identificar en las ciudades del Altiplano central por las características propias de la densidad y cantidad de población. El altepetl constituye el elemento clave que define el territorio y que, a su vez, limita el ámbito de influencia entre el centro y su periferia. Sin embargo, en los últimos años se ha incorporado la idea del Estado segmentario para el Altiplano central, lo que obliga a la redefinición del altepetl en lo que se refiere a control territorial y definición de fronteras (Hirth 2012: 72-73). Para Hirth, lo que definía al altepetl no eran tanto las fronteras territoriales cuanto las relaciones sociales entre señores y subordinados (Hirth 2012: 85-86). En relación con las unidades políticas que se describen en las fuentes históricas, habría que sumar a la extensión del altepetl factores tales como la incorporación de nuevas comunidades por conquista, migración o asimilación. Este es un proceso lento y con particularidades propias, pero alrededor del siglo XIV se pueden definir numerosos centros urbanos compuestos por una sociedad que, por la densidad creciente del patrón de asentamiento y por la especialización de los centros urbanos y sus habitantes, tiene ya las características que denotan un verdadero Estado o ciudad-Estado en los términos de Smith y Hodge (1994: 11).

Este proceso de constitución de un Estado se dio en tiempos diversos dependiendo de las casuísticas de cada altepetl. Para Schroeder (1994: 186), aparece cuando un grupo migratorio se transforma en sedentario; el autor hace de ese momento la fecha de inicio de la correspondiente entidad política. Por lo tanto, cada altepetl ha de ser analizado cuidadosamente para determinar el paso de una sociedad tribal a un cacicazgo y, posteriormente, a la construcción de un Estado; ha de considerarse en qué manera ese paso se corresponde con la transformación de una sociedad con alta movilidad a otra sedentaria, así como determinarse si hubo cambios intermedios. Como ejemplo, para los mexicas, Zantwijk (1975) considera que existen cuatro etapas en su formación, que abarcan desde la salida de Aztlan hasta la conformación de la Triple Alianza y el gobierno de Ahuitzotl ${ }^{5}$. Una propuesta más global para determinar los momentos claves de la caracterización de un altepetl pone el énfasis en: a) su periodo de migración; b) el momento de establecimiento en un territorio específico con la fundación del altepetl; c) el periodo de constitución y consolidación como entidad

\footnotetext{
${ }^{4}$ Siguiendo la idea de «volver a lo básico» (back to basics que dirían los colegas estadounidenses) y siguiendo la tradicional caracterización de Service sobre lo que se determina para el Estado: clases sociales, ejército, una religión formal, normas jurídicas, figuras institucionalizadas y un ejército (Service 1975: 33).

5 Para este autor, la sociedad mexica se desarrolla es cuatro etapas que van desde la constitución de una sociedad tribal, a un cacicazgo cuando se instalan en Chapultepec y posteriormente constituyéndose como un estado incipiente como aliados con Azcapotzalco y llegando a ser un estado consolidado con la instauración de la Triple Alianza.
} 
sociopolítica; y d) los elementos étnicos constituyentes con los que se identifican (mitos de origen, religión, territorio) (García 2007).

Estos altepeme, que funcionan como capitales y que son históricamente conocidos en el Posclásico Medio y Tardío, tenían las características que permiten definirlos como Estados; sin embargo, difieren en su constitución étnica, demográfica y territorial. Las unidades más débiles y menos cohesionadas serán conquistadas o anexionadas por los altepeme más poderosos de Azcapotzalco y posteriormente por Tenochtitlan. Según Hodge (1984), después de la caída de Tula se formaron en la Cuenca de México alrededor de 60 unidades políticas en donde: «El sinónimo altepetl está asociado con la idea de gobierno, así como con la de territorio (...) una ciudad con su territorio adyacente, gobernada por un tlatoani o rey, fue la unidad política básica náhuatl, que los españoles llamaron señorío» (Hodge 1984: 17).

En ese mismo trabajo, Mary Hodge menciona algunos asentamientos significativos para el Posclásico, como son: Azcapotzalco (tepaneca); Texcoco, Coatlinchan, Huexotla (aculhua); Chalco, Tlalmanalco, Amecameca, Tenango, Chimalhuacan (Confederación Chalca); Culhuacan (culhua-tolteca); Xochimilco (xochimilca); Cuautitlan (cuautitlahneca); Xaltocan (xaltocameca); y Tenochtitlan y Tlatelolco (mexicas). Dicha autora considera que, para el Posclásico Medio, algunos de ellos se encontraban unidos étnicamente, formando, a su vez, confederaciones de varios altepeme $^{6}$. En este sentido, podemos ver que en algunos casos, como en ChalcoAmaquemecan, había al menos seis ciudades-Estado, cada una con un tlahtoani, correspondiendo a diferentes grupos étnicos (Shroeder 1994).

\section{El desarrollo del altepetl durante el Posclásico Temprano (1150-1325)}

Lo que dificulta el análisis no es tanto la consideración de si se puede definir a los altepeme como Estados, sino las diferentes sutilezas en la integración sociopolítica de los mismos en un momento histórico determinado. En el caso del periodo Chichimeca (1150-1325), la conjunción de las fuentes históricas y los datos arqueológicos son determinantes para intentar comprender factores como la integridad, la cohesión, el tamaño y la expansión de cada uno de los centros mencionados en los textos. Davies advierte de la complejidad de definir lo chichimeca dada la multiplicidad de significados del propio término (Davies 1980: 79). En términos generales, se puede decir que existe un patrón de desarrollo que puede ser descrito en las siguientes etapas: a) migración; b) establecimiento y fundación del altepetl; c) consolidación del asentamiento y expansión territorial de los sitios adyacentes; d) establecimiento de alianzas; e) guerras de conquista /expansión territorial; f) formación de entidades políticas mayores (García 2007).

Este patrón de desarrollo no constituye una lista de niveles que hayan de considerarse de manera secuencial, ya que alrededor del siglo XIII, mientras que algunos grupos chichimecas de la Cuenca de México -como los acolhuas, tepanecas o chalcas- se pueden considerar como Estados, otros - como los mexicas- tenían una estructura sociopolítica menos compleja. Aun más, no hay que olvidar que el valle

\footnotetext{
${ }_{6}$ Con esto se quiere decir que existía en cada confederación un grupo étnico mayoritario y hegemónico; no obstante, sabemos que los altepeme eran conglomerados pluriétnicos. Véase el trabajo de Schroeder (1994) sobre este tema, donde se trata la conformación multiétnica de Chalco-Amaquemecan.
} 
de Teotihuacan para estas fechas ya no tiene la cohesión política y territorial que mantuvo en fases precedentes (Braniff 2005; Crider et al. 2007). Según las fuentes históricas, el periodo de migración de estas sociedades se inició desde comienzos del siglo XII o un poco antes y se dio a lo largo de unos 150 o 200 años, por lo que su desarrollo, a partir de su establecimiento definitivo, fue también desigual. Estos grupos migratorios tal vez no tuvieron un mismo origen o una misma auto-identificación étnica, ya que en las fuentes más antiguas son conocidos como chichimecas (Alva Ixtlilxochitl 1977; Códice Xolotl 1980; Mapa Quinatzin 1891; Torquemada 1975) y en las más recientes, que se refieren a la migración mexica ${ }^{7}$ (Durán 1995; Códice Ramírez 1985; Códice Boturini 1975; Códice Aubin 1980; Códice Azcatitlan 1995), y en Clavijero (1991: 63) se les denomina nahuatlacas, refiriéndose «a siete tribus de una misma nación». Todos los relatos dan la imagen de que, a la llegada de los grupos chichimecas a la Cuenca de México, se encontraron con un territorio poco poblado con excepción de los antiguos asentamientos toltecas del sur de la región. Según Alva Ixtlilxochitl, los chichimecas que llegan en esta primera oleada serían sociedades con similares niveles de organización sociopolítica, pero con una gran variabilidad interna. Sin embargo, y en su mismo relato, reconoce la existencia de grandes señores que provenían de un «reino» y constituyendo una «nación chichimeca» (Alva Ixtlilxochitl 1977: 291-292). Como en todos los relatos, hay que matizar aspectos tales como el propio contexto de la fuente histórica y los datos arqueológicos no sustentan la idea de una migración masiva. Posiblemente aquí se encuentre uno de los ejemplos de la alteridad en los textos históricos mencionada anteriormente. A pesar de que Zantwijk considera a estos grupos como socialmente complejos, no podemos considerarlos, bajo los términos de Mary Hodge, como Estados ${ }^{8}$ (Hodge 1984: 2; 1997; Zantwijk 1975: 11).

Entramos, por lo tanto, en un ámbito complejo de fronteras difusas para definir la estructura sociopolítica de estos grupos chichimecas, en el que cabría considerar las investigaciones de Griselda Sarmiento sobre las sociedades tribales jerárquicas ${ }^{9}$ (Sarmiento 1992: 80) y, sin duda alguna, retrotraernos a las definiciones más clásicas y fundadoras del campo de estudio, con lo que Morton Fried (1960: 719) definió como sociedades de rango y Elman Service (1971: 133-134) como cacicazgos o jefaturas ${ }^{10}$. Nos encontramos en ese momento de la estructura social en la que la figura del jefe trasciende el simple liderazgo personal, institucionalizándose a través de su carisma y habilidades personales para planear y organizar el trabajo comunitario (Service 1971: 140). Para consolidar y, sobre todo, poder transmitir a sus allegados

\footnotetext{
7 En algunas fuentes mexicas, se habla de la migración desde Aztlán y de un número variable de grupos o tribus que algunas veces son siete u ocho grupos. De cualquier manera, creemos que este asunto no está suficientemente investigado, pues la arqueología demuestra en muchos de los casos que antes de la fecha de llegada de esos grupos, ya existían poblaciones en lugares como Xochimilco, Cuitlahuac o Chalco, lugares donde la aparición de la cerámica Azteca II demuestra que las poblaciones eran más antiguas de lo que mencionan las fuentes.

8 Mientras Zantwijk sugiere una organización clánica fuertemente jerarquizada, Hodge pone el énfasis en un gobierno centralizado y firmemente arraigado en el territorio que no se reconoce para los primeros grupos chichimecas (Hodge 1984: 2; Zantwijk 1975: 11).

9 «(...) es un tipo social que no presenta todavía una división social en clases, pero tampoco es una sociedad igualitaria ya que existe cierta jerarquía entre los individuos, característica que la diferencia de las sociedades tribales» (Sarmiento 1992: 80).

${ }^{10}$ «(...)...el surgimiento de los cacicazgos parece haber estado relacionado con una situación totalmente ambiental, la cual indujo a la especialización en la producción y a la redistribución de ésta, desde un centro de control» (Service 1971: 133-134).
} 
el poder, se crearán una serie de mecanismos ideológicos con los que poder conservar, tanto su linaje como sus agremiados, el estatus y las prebendas que se derivan de éste (Flannery 1972: 17). La consolidación de este modelo será la herencia por derecho de primogenitura, partiendo del ámbito de lo familiar hasta convertirse en el mecanismo de transmisión del poder y del gobierno del altepetl. Este elemento será clave en la evolución posterior de las jefaturas a Estados durante el Posclásico. Algunas de las fuentes históricas más importantes que se refieren a este momento del Posclásico Medio - como son el Códice Xolotl, el Mapa Quinatzin, el Mapa Tlotzin (1885), Torquemada, Alva Ixtlilxochitl o Veytia- mencionan que los chichimecas eran cazadores-recolectores vestidos con pieles que, al llegar a la Cuenca de México, fundan la Chichimecatlalli. Xolotl es en este sentido el padre fundador de este «imperio». Para Davies (1980), esta última acepción no es compatible con los primeros momentos de la sociedad del Posclásico Medio, ya que la gestión de grandes territorios requiere de un aparato burocrático bien consolidado y jerarquizado que no coincide con el modelo que tenemos de los primeros grupos chichimecas ${ }^{11}$.

En este primer escenario histórico, se debería aceptar el hecho de que las sociedades del Posclásico Medio en la Cuenca de México fueron sociedades jerárquicas que, al establecerse, inician un rápido proceso de evolución política cristalizado al principio en un asentamiento permanente o altepetl, en el sentido de unidad sociopolítica que integra y controla un territorio. Los primeros asentamientos, como Tenayuca, Azcapotzalco, Texcoco, Chalco, Xaltocan y Chalco, derivarán progresivamente en centros urbanos (Códice Xolotl 1980; Schroeder 1994: 182-192).

\section{La caracterización de las sociedades chichimecas desde la jefatura/cacicazgo hasta el Estado}

Los párrafos siguientes tienen como meta sistematizar el proceso de transformación social, política y económica de las sociedades chichimecas con objeto de comprender su evolución a lo largo del Posclásico Medio. La sistematización se concreta en cinco fases o pasos en la transformación de las jefaturas en Estados, considerando los factores relevantes desde la llegada y asentamiento de los primeros chichimecas en la Cuenca de México hasta la consolidación y sostenimiento del modelo sociopolítico en los años previos a la hegemonía mexica. No ha de entenderse como una línea rígida que obligatoriamente hubieran de seguir los diferentes casos, sino como un primer acercamiento a una sistematización que pueda servir de base para caracterizar la diversidad del asentamiento chichimeca a lo largo del Posclásico.

1. La cuestión de la fundación del asentamiento principal (altepetl) es un tema abordado en todas las fuentes, tanto como parte de un evento con características épicas y mitológicas como detallando la pertenencia étnica, el nombre de sus líderes, el lugar de procedencia y su deidad principal (Schroeder 1994: 186-187). La existencia de un relato fundacional no implica en sí mismo la existencia real de ese asentamiento primordial ex novo (altepetl), pudiéndose dar una refundación, reocupación o asimilación en un área con asentamientos previos. La creación de un altepetl implica

\footnotetext{
${ }^{11}$ Sin embargo Davies (1980: 87) dice: «Yo estoy convencido que cualesquiera Chichimecas, que participaron en la destrucción de Tula Xicocotilan y quienes invadieron el Valle de México, estuvieron bien acaudillados y altamente organizados y tuvieron poco en común, por decir, con los guamares de tiempos de la conquista».
} 
también una delimitación del área de influencia del mismo y, por lo tanto, una toma de posesión del territorio aledaño, reconociéndose como parte del mismo paisaje o reinventando un nuevo paisaje cultural incorporando los mitos de origen. Es un paso clave y altamente simbólico ya que constituye la sedentarización del grupo y marcará la relación con sus contemporáneos. A partir de ese hecho, la sociedad inicia otros procesos (demográficos, económicos, políticos) que a la larga convergen en la aparición de la sociedad estatal.

2. La cuestión de las tierras entre las elites (repartimientos, apropiaciones, pactos). El estudio de la propiedad de la tierra es un tema de gran importancia, pero de muy difícil análisis para todo el periodo prehispánico. Gracias a algunas fuentes documentales se pueden establecer algunas líneas generales de cómo se representa el repartimiento de tierras entre los personajes principales ${ }^{12}$ (Códice Xolotl: Plancha II; Veytia 1979: 277; Durán 1995). Asimismo, en la Plancha I del Códice Xolotl se menciona que el caudillo chichimeca repartió el territorio de la Cuenca de México entre tres jefes a quienes casó con sus hijas (Coatlinchan, Azcapotzalco y Xaltocan), mientras que a su hijo Nopaltzin le dio Texcoco. La posibilidad de que un recién llegado a la Cuenca como Xolotl estuviera en disposición de repartir tierras a sus allegados y familiares nos abre de nuevo varias preguntas vinculadas a la jerarquía política, el ejercicio del poder y a la manera en que se percibe el mismo. El altepetl se concibe como un elemento en expansión derivado del crecimiento demográfico y de la progresiva apropiación de tierras que tiene como resultado, en este caso, la existencia de nuevos altepeme vinculados con el originario, pero a su vez con cierta autonomía política. A la larga, esta acción tuvo como consecuencia la creación de una estructura territorial jerarquizada, derivada del grado de parentesco del gobernante local con el gobernante principal del altepetl $^{13}$. Por ejemplo, se puede ver en el Códice Xolotl, en la Plancha III (Figura 1), la sucesiva llegada de los grupos chichimecas y cómo Xolotl hace la repartición y división del territorio. La tercera acción es la repartición de un número de vasallos, que eran los mismos que venían con cada uno de los grupos migratorios. Estos vasallos eran los que mantenían a la nobleza a través del tributo local (Durán 1995: Cap. V; Veytia 1979: 287) ${ }^{14}$.

3. La consolidación de este modelo y su expansión supuso la creación de estas nuevas comunidades que ocuparon tierras con potencial agrícola, acceso a aguas o espacios de frontera. Los lazos de unión se vincularon con el parentesco, pero eso no implica una relación igualitaria, sino disimétrica en relación con el estatus, el género y la vinculación más o menos directa con el linaje principal del altepetl primigenio.

\footnotetext{
${ }^{12}$ Quizás una de los mejores ejemplos de esto es la relación que da Durán (1995, Cap. 5: 93) «Habló Vitzilpochtli á su sacerdote ó ayo y díxole: dí a la congregación mexicana que se dividan los señores cada uno con sus parientes amigos y allegados en quatro barrios principales, tomando en medio a la casa que pare mi descanso aueis edificado; y que cada parcialidad edifique en su barrio a su voluntad».

${ }^{13}$ Claessen (1979: 97) ha mencionado la importancia del Clan Cónico (Kirchhoff 1968) a propósito del significado que tiene el parentesco en las relaciones políticas en una sociedad cacical, en donde «la característica predominante del clan cónico es que los segmentos y los individuos son arreglados jerárquicamente con base a su antigüedad genealógica, es decir por orden de nacimiento. Dentro de cada linaje, los individuos son colocados de acuerdo a la distancia que guardan con respecto al fundador del linaje y los diversos linajes, a su vez, son colocados de acuerdo con la posición que tiene cada fundador de linaje en la genealogía del clan».

${ }^{14}$ En este apartado nos referimos a lo que Mohar (1987: 14) define como tributo local: «El tributo se define básicamente por el pago de bienes y servicios (...). Este se daba a nivel local, por el pueblo común para el mantenimiento de sus señores o gobernantes nativos. Consistía básicamente, de servicio en casa de los señores, el trabajo de sus campos y la provisión de alimentos, agua, leña, etcétera».
} 


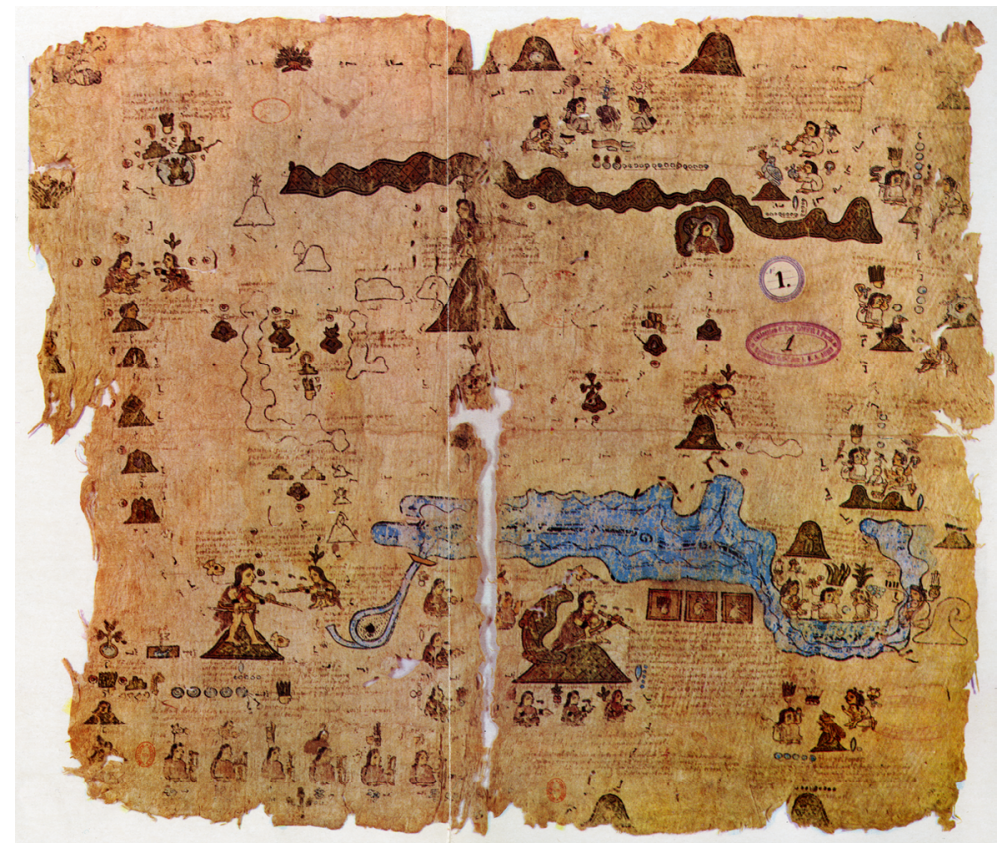

Figura 1. Plancha II del Códice Xolotl (1980): asentamiento, repartición y delimitación del territorio por parte de los chichimecas.

Alva Ixtlilxochitl (1977, I: 321) menciona que había 27 reinos que estaban bajo el mando de Techotlalatzin, gobernante chichimeca (tataranieto de Xolotl), los cuales incluían a las principales ciudades de la Cuenca de México (Azcapotzalco, Xaltocan, Xochimilco, Chalco, etc.) y sus sitios subordinados. De acuerdo con este texto, se pueden inferir entre cuatro y cinco tipos de localidades por su diferente tamaño y población. Sin embargo, hay que considerar que uno de los reinos (supuestamente) subordinados era Azcapotzalco, que a su vez tenía bajo su poder a casi todas las localidades del poniente de la Cuenca, como Tlacopan, Atlacuihuayan, Tenayuca, Tultitlan y Coyoacan ${ }^{15}$. A su vez estas localidades debieron de tener como sujetos suyos a sitios más pequeños, la mayoría de los cuales para este periodo no están mencionados en las fuentes, pero es común encontrar restos arqueológicos en el área urbana de la Ciudad de México que demuestran que existieron pequeños asentamientos, los cuales seguramente estaban subordinados a los conocidos históricamente. En este caso se observa claramente un gradiente de cuatro a cinco tipos diferentes de asentamientos, desde la capital imperial constituida por Texcoco, pasando por la capital del altepetl regional, como Azcapotzalco, y siguiendo con los sitios de tercer orden, como Tlacopan, Tultitlan o Coyoacan, hasta sitios más pequeños como Atlacuihuayan (ver también Hodge 1984).

\footnotetext{
${ }^{15}$ Más bien aquí parece que el historiador texcocano, daba preponderancia al altepetl de Texcoco, pero de la lectura de otras fuentes, se infiere que quizás las fuerzas entre acolhuas y tepanecas eran similares y que el sojuzgamiento se dio hasta la época de Xolotl e Ixtlilxochitl.
} 


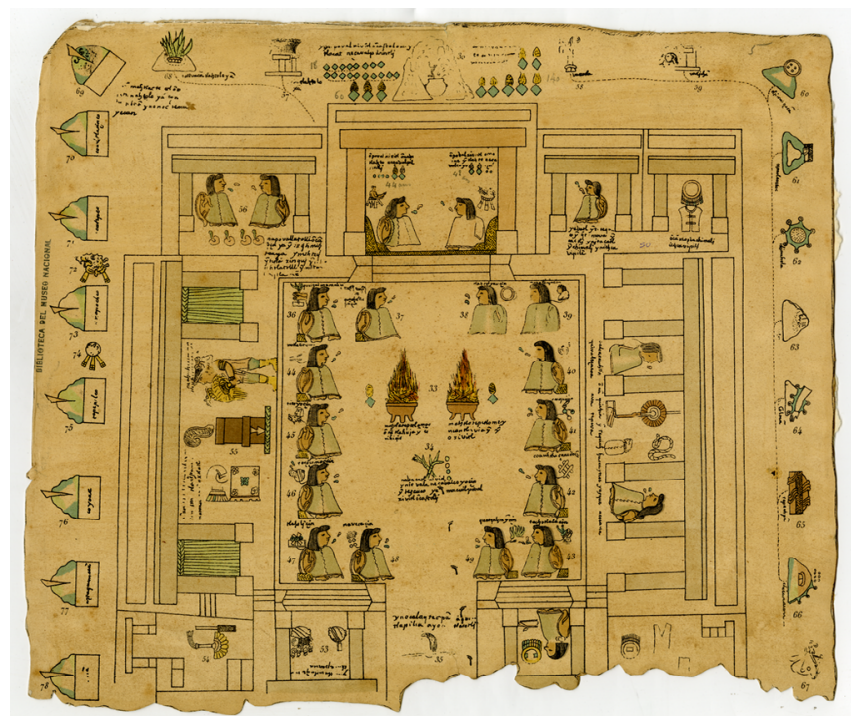

Figura 2. Mapa Quinatzin, Plancha II, Tecpan de Texcoco (Mapa Quinatzin 1891: lámina 212).

4. La política de pactos y alianzas es clave para la consolidación de este modelo y es consecuencia de la expansión demográfica. Las relaciones de dependencia pueden tener también origen en el tipo de relaciones de intercambio. Las alianzas matrimoniales son cruciales para comprender el grado y la importancia de las relaciones entre los altepeme, operando como un mecanismo para vincularse por lazos familiares y entre grupos. Se ha utilizado, así mismo, el concepto de confederaciones de tipo étnico, el cual necesitaría un estudio más detallado por lo que implica el uso de la etnicidad como un factor determinante. Un ejemplo que abogaría por este concepto, pero referido a una fase más tardía, se puede observar en la Plancha II del Mapa Quinatzin (Figura 2), en donde se observa el tecpan de Texcoco, a manera de edificio que cumple con funciones estatales, en el que se representan todas las ciudades aliadas del altepetl del Acolhuacan (Mohar 2004). En Pomar (1975: 69) se hace una resumida relación del tecpan de Texcoco y de sus dimensiones, así como de la importancia de este edificio como «palacio real» y se resalta su importancia como el edificio de residencia de los gobernantes. Los glifos emblema de la confederación Aculhua posiblemente estaban labrados y colocados sobre algún friso, constituyendo elementos importantes y visibles. Resaltan en ese documento todas las características que le daban al edificio el carácter de «Palacio Real» (Manzanilla 2001; Mohar 2004: 239).

5. Procesos de cohesión y procesos de desintegración: del pacto al conflicto. En términos generales, se conocen poco los procesos de violencia y conflicto en el centro de México, sobre todo en los siglos previos a la hegemonía mexica. La historiografía menciona guerras entre los diferentes señores, pero lo cierto es que existen pocas evidencias de conflicto. Los indicadores arqueológicos, como serían los cambios en el patrón de asentamiento y la presencia de incendios, murallas y fortificaciones, permanecen esquivos. Los motivos de los conflictos debieron de ser variados, pero 


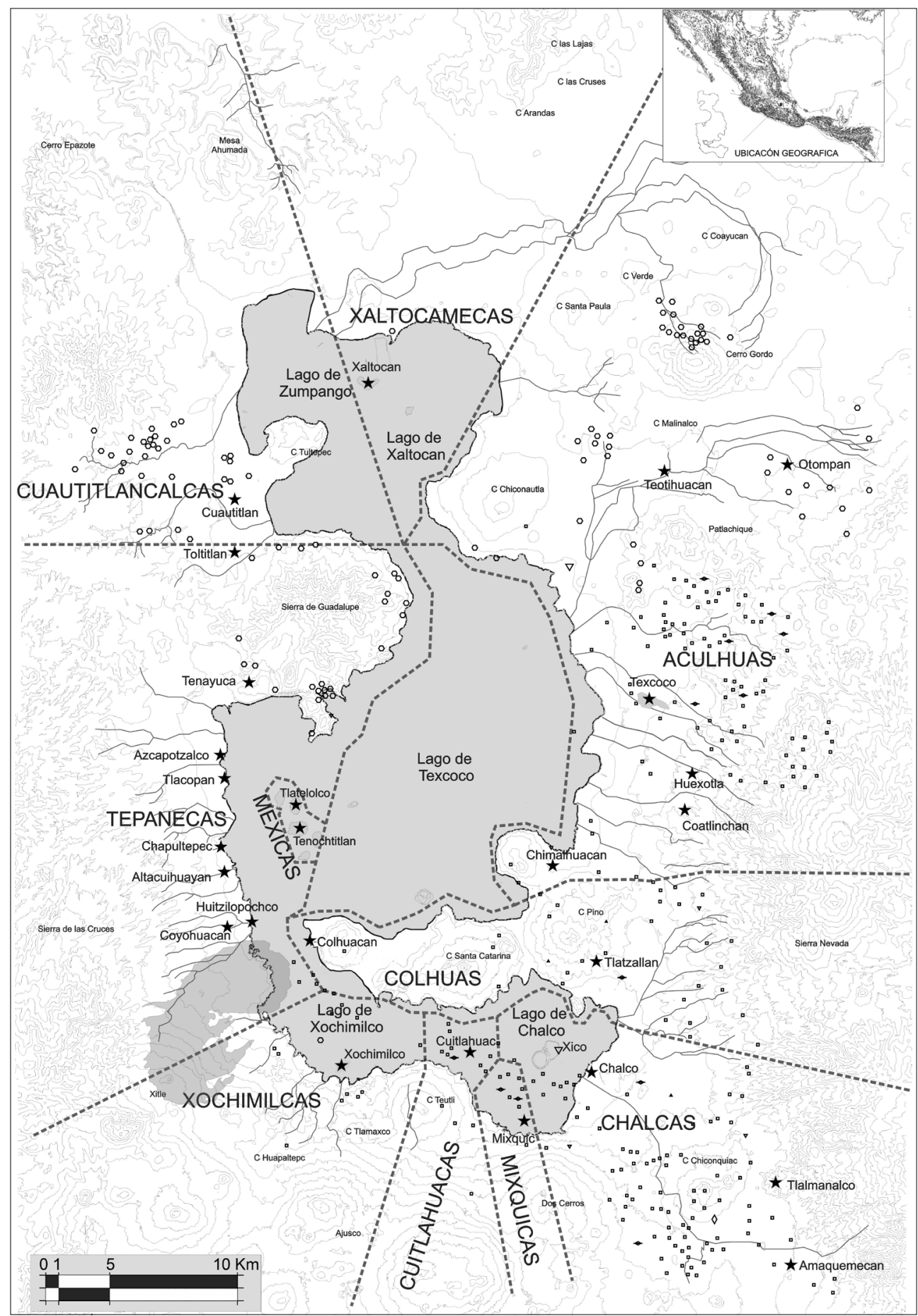

Figura 3. Posibles unidades políticas durante la fase Azteca II, alrededor del año 1400 d.C. (Plano realizado por Ulises Santa Cruz Rendón). 
el tributo y la acumulación de poder y prestigio debieron de ser cruciales. Esto es el resultado del rompimiento del equilibrio de fuerzas entre varios altepeme y parece una consecuencia de los anteriores procesos. Inicialmente, los asentamientos de la Cuenca no tuvieron la capacidad para enfrentarse entre sí, pero, como se ha visto, los procesos de crecimiento poblacional y la formación de las entidades políticas regionales (aculhuas, tepanecas, chalcas, cuitlahuacas, mixquicas, xochimilcas, culhuacanos, xaltocamecas, cuautitlanecas), estructuradas ya como Estados, encontraron en la expansión territorial un mecanismo para fortalecerse. En consecuencia, el incremento del militarismo y la consolidación de un ejército mucho más estructurado que en fases anteriores hubieron de ser significativos en la constitución del ejército mexica. El ejemplo más palpable de esto fue la guerra entre Azcapotzalco y Texcoco, que se ha interpretado como muestra del conflicto por la hegemonía territorial utilizando como pretexto el derecho dinástico y la legitimación en la sucesión en el poder (Alva Ixtlilxochitl 1977: 40); en el caso del gobernante de Texcoco, Ixtlilxochitl (el padre de Nezahualcoyotl) procedía por línea directa del fundador de la dinastía Xolotl y, por su parte, Tezozomoc, gobernante de Azcapotzalco, era nieto de Xolotl, ya que su madre fue hija de éste ${ }^{16}$. Sin embargo, lo que resulta de mayor interés es que será a partir de ese momento cuando se observa un proceso de conflictos entre diversas unidades territoriales que tendrán como consecuencia la creación de entidades mayores, las denominadas confederaciones de Azcapotzalco (tepaneca) y de Texcoco (acolhua). Algunos autores consideran que es entonces cuando se crean las bases de un sistema imperial (Carrasco 1984: 73), en la época en que Tezozomoc conquistó Texcoco y las ciudades aliadas aculhuas (Alva Ixtlilxochitl 1977, II: 80). Un ejemplo de estos cambios y continuidades se observa en el denominado Imperio Tepaneca. Según las fuentes, el control dependía de la repartición del territorio conquistado entre los herederos del tlatoani y miembros destacados de las elites (Carrasco 1984: 74); con este sistema se controlaba el territorio y se evitaban sublevaciones mientras se mantuviera el statu quo en la Cuenca de México. El surgimiento de dos fuerzas emergentes con aspiraciones territoriales (Tenochtitlan y Tlatelolco) y la formación de la llamada Triple Alianza como una entidad sociopolítica superior será una continuación del proceso de formación de los altepeme en el Posclásico Temprano y la culminación del desarrollo estatal (Figura 3).

El Cuadro 1 condensa una primera imagen de este proceso histórico y arqueológico como una herramienta indicativa que nos permita poder seguir avanzando en su conocimiento.

\footnotetext{
${ }^{16}$ En este conflicto entre ambas potencias, se aducen como pretextos la legitimidad de las líneas de sucesión dinástica, pero la verdadera esencia del conflicto es la lucha por la toma de decisiones en las altas esferas políticas y que tienen que ver directamente con las cuestiones de orden económico.
} 


\section{Cuadro 1. Propuesta de esquema del cambio sociopolítico durante el Posclásico en la Cuenca de México}

\begin{tabular}{cccc}
\hline Característica & $\begin{array}{c}\text { Tribu chichimeca } \\
\text { (etapa de migración) }\end{array}$ & $\begin{array}{c}\text { Jefatura chichimeca } \\
\text { (etapa del primer } \\
\text { asentamiento) }\end{array}$ & $\begin{array}{c}\text { Estado chichimeca } \\
\text { (formación y consolidación } \\
\text { del Estado) }\end{array}$ \\
\end{tabular}

Tipo de No existía el altepetl pues Se funda el altepetl. Este asentamiento el asentamiento es esta- hecho constituye uno de cional; en las diferentes los momentos más imporhistorias se habla de que los tantes, pues el establecigrupos migratorios se tras- miento definitivo trae como ladaron de un lugar a otro consecuencia una serie de cambios a nivel estructural en todos los grupos $\begin{array}{ll}\text { Tipo de } & \text { No hay un territorio defi- } \\ \text { territorio } & \text { nido }\end{array}$
El asentamiento se urbaniza, consolidándose y construyéndose edificios específicos como la plaza, el templo principal, el palacio, el juego de pelota y el área de mercado

Se definen los linderos de los altepetl. Desde la etapa anterior se observa un proceso en el cual pudo haber solapamiento de territorios, que posiblemente condujo a la anexión de los altepetl más pequeños a través de la conquista, pero también se observa que algunos altepetl se convierten en vasallos o protegidos de los altepetl más grandes a través de alianzas matrimoniales

Adaptación al territorio

La población está adaptada a condiciones de vida migratoria

La población está adaptada a condiciones de vida sedentaria

Durante este periodo los asentamientos principales (los más antiguos) sufrieron una transformación, convirtiéndose en centros urbanos, por lo que el modo de vida cambió, desarrollándose una adaptación por parte de la población a ese modo de vida urbano; surgen así una gran cantidad de "especializaciones productivas urbanas"

$\begin{array}{ll}\text { Recursos } & \text { Hay poca presión sobre } \\ \text { alimenticios } & \text { los recursos alimenticios. } \\ & \text { Empieza a desarrollarse un } \\ & \text { periodo con mucha presión } \\ & \text { sobre los recursos alimen- } \\ & \text { ticios }\end{array}$

Comienza a desarrollarse La presión sobre los recursos un periodo de presión sobre es tan grande que se crean los recursos naturales, por lo que los chichimecas comienzan a sembrar y a cuidar los recursos de caza y recolección.

sistemas de producción intensiva y el área de la Cuenca se "especializa" en diversos productos. La conquista de unos altepeme por otros tiene como consecuencia que se entregue tributo en forma de recursos alimenticios

Con la transformación de los asentamientos en sitios urbanos, se crea una gran cantidad de oficios y especializaciones productivas; los especialistas abandonan el sector primario para dedicarse a actividades productivas a tiempo completo 
Intercambio Existe poco intercambio, pues la producción sólo satisface las necesidades inmediatas del grupo, por lo cual el intercambio se debió realizar a una escala mínima

Inicialmente existe muy poco intercambio, pero al desarrollarse e incrementarse la producción empieza un periodo de intercambio a escala de la Cuenca de México

Composición étnica y nombre del grupo

El grupo migratorio está compuesto por varios grupos étnicos, pero existe un componente étnico mayoritario que lidera al grupo

\section{Demografía}

La población no crece y mantiene tasas restringidas de crecimiento poblacional

Después del establecimiento y como consecuencia de "mejores condiciones", la población crece a un ritmo continuo, superando los números del asentamiento inicial

El altepetl se funda por uno o varios componentes étnicos pero existe un componente étnico mayoritario que le otorga el nombre al altepetl
Con la especialización productiva, se crean los primeros mercados locales que satisfacen la demanda de productos a nivel del altepetl y sus comunidades satélites. Con el tiempo, se crearon en los principales huey altepetl mercados regionales donde el intercambio se realizaba a nivel mesoamericano (v.gr. el mercado de Tlatelolco)

Está plenamente demostrado que los altepetl eran asentamientos multiétnicos, existiendo una variedad de formas. Con el crecimiento de los asentamientos y su transformación en urbes, nuevos componentes étnicos se fueron incorporando, haciendo de estos sitios lugares multiculturales

Es posible que después de la etapa inicial de establecimiento definitivo, los asentamientos fueran creciendo a un ritmo continuo y esta tendencia fue irreversible. Asentamientos iniciales, tal vez con algunos cientos de habitantes, se convierten en ciudades del orden de decenas de miles a la llegada de los españoles

En la etapa del altepetl desarrollado, la religión se institucionaliza como religión de Estado. Los dioses tutelares originarios son los dioses dominantes del panteón mesoamericano. Se creó así todo un gradiente jerárquico de sacerdotes $\mathrm{y}$ una "burocracia" religiosa. Se crearon nuevos cultos y rituales para satisfacer una religión de masas. Con el advenimiento de la sociedad dividida en clases sociales, se creó un culto estatal, cuyo correlato más conocido es el culto a Huitzilopochtli de los mexicas

Después del asentamiento inicial, se desarrollaron grupos armados que se convirtieron en una casta militar profesional que tenían la función de organizar ejércitos para tiempos de guerra. Este grupo de guerreros profesionales que dirigían los ejércitos, formaron parte de la clase alta, teniendo privilegios especiales otorgados por el gobernante de turno 
Tipo de líder En las etapas tribales se designaba a un líder carismático, que actuaba para tomar las decisiones; se le designaba como cuauhtlato cuya traducción es "un noble que habla"
Estructura política
Una vez que los grupos se asientan definitivamente con el tiempo, los cuautlatos se convierten en tlathtoani
La estructura política no rebasa el nivel de la tribu con un grupo de hombres de experiencia que toman las decisiones. En esta época podría haber surgido la figura de un jefe carismático que se elige por sus características para conducir al grupo; el mando, pues, se ejerce por liderazgo
Se puede formar una estructura política de clan cónico y con el tiempo se forman varios clanes, relacionados con un linaje principal, que van ocupando el territorio a medida que el altepletl crece. El jefe principal del altepletl es descendiente de los primeros jefes y sus parientes cercanos son elegidos para desarrollar los principales puestos en la estructura tribal. De acuerdo con esto, el poder se ejerce y se hereda entre la familia principal del altepletl y en cada caso del grupo étnico hegemónico
Las viejas estructuras del cacicazgo desaparecieron paulatinamente con el advenimiento y la creación de instituciones gubernamentales, es decir, una burocracia de Estado necesaria para controlar y administrar el complejo sistema de gobierno. Esta estructura, que se inició con la designación de familiares cercanos a los jefes tribales para el desarrollo de las funciones del gobierno, con el tiempo devino en un aparato donde ya no importaba ser un personaje cercano a las familias de los altos dignatarios, pues los funcionarios se designaban por sus méritos y podían ser sustituidos en cualquier momento por el tlahtoani

Con el tiempo los tlahtoani, también cambiaron de status, convirtiéndose en Huey Tlahtoani o gobernante supremo de una confederación de altepeme. Los tlahtoani se diseminaron por todos los asentamientos de menor jerarquía. Los tlahtoani residían en un tecpan o palacio, existiendo también el Huey Tecpan, que era el palacio donde habitaban los monarcas máximos como Moctezuma o Nezahualcóyotl. El desarrollo del cargo de tlahtoani está cercanamente asociado con el desarrollo de la arquitectura palaciega del Posclásico

\section{Conclusiones}

La arqueología de la Cuenca de México se ha considerado tradicionalmente como una serie encontrada de procesos de homogeneización política y social y procesos de disgregación. Sin duda alguna, la tradicional subdivisión temporal de carácter tripartito y secuencial entre Preclásico, Clásico y Posclásico ha contribuido a dicha percepción, tanto en el ámbito académico como en el de la difusión. Sin embargo, para el estudio de determinados procesos complejos de dinámicas políticas y sociales entre las poblaciones prehispánicas, los periodos de transición, intermedios, de balcanización o de disgregación, nos ofrecen posibilidades valiosas para comprender las dinámicas culturales de distintas sociedades en un entorno cambiante. Si bien el término contacto parece remitirnos al momento de la llegada de los españoles al continente americano, lo cierto es que las sociedades indígenas debieron de asumir sus propios momentos de contacto cultural entre grupos que, aun compartiendo algunos 
elementos culturales, los plasmaron política y socialmente de maneras muy distintas. Por otra parte, comprender los elementos relevantes de los procesos de integración de grupos humanos, aparentemente menos complejos políticamente, con los herederos de sociedades con una larga tradición urbana, es una cuestión que hoy en día resulta de actualidad atendiendo a los fenómenos migratorios contemporáneos.

El altepetl se erige en el elemento clave, que puede ser estudiado desde la Arqueología y la Historia, atendiendo a las peculiaridades tanto del objeto de estudio como de las propias disciplinas que lo analizan. En estas páginas se ha abordado un primer acercamiento metodológico, considerando algunos puntos esenciales para conocer mejor las bases conceptuales del fenómeno político-territorial durante el Posclásico. Aun existiendo diferentes definiciones y acercamientos a la cuestión y a pesar de no ser posible asumir la presencia de un altepetl prototípico, aquí se han trazado cinco fases o pasos con los cuales caracterizar los procesos de conformación del mismo sobre la base de fuentes históricas y arqueológicas y de las teorías antropológicas. Ello permite avanzar en la interpretación y establecer unas primeras pautas para el estudio del fenómeno del altepetl en la Cuenca de México durante el periodo Chichimeca

\section{Referencias}

Alva Ixtlilxochitl, Fernando de

1977 Obras Históricas: incluyen el texto completo de las llamadas Relaciones e Historia de la nación Chichimeca en una versión establecida con el cotejo de los manuscritos más antiguos que se conocen..., edición de Edmundo O’Gorman. México: Instituto de Investigaciones Históricas, Universidad Nacional Autónoma de México.

BRANIFF, Beatriz

2005 «Los chichimecas a la caída de Teotihuacan y durante la conformación de la Tula de Hidalgo», en Reacomodos demográficos del Clásico al Posclásico en el centro de México, Linda Manzanilla, coord., pp. 45-56. México: Instituto de Investigaciones Antropológicas, Universidad Nacional Autónoma de México.

BRUMFIEL, Elizabeth M.

1976 Specialization and Exchange at the Late Postclassic (Aztec) Community of Huexotla, Mexico. Tesis doctoral. Dep. of Anthropology, University of Michigan.

Carrasco Pedro

1984 «The Extent of the Tepanec Empire», en The Native Sources and the History of the Valley of Mexico, Jacqueline de Durand-Forest, ed., pp. 73-93. Oxford: BAR International Series 204.

1996 Estructura politico-territorial del Imperio tenochca. México: Fondo de Cultura Económica - El Colegio de México.

CARrasco, Pedro y Johanna Broda (eds.)

1976 Estratificación social en la Mesoamérica prehispánica. México: SEP-Instituto Nacional de Antropología e Historia.

1978 Economía política e ideología en el México prehispánico. México: Editorial Nueva Imagen - Centro de Investigaciones Superiores del Instituto Nacional de Antropología e Historia. 
Caso, Alfonso

1967 Los calendarios prehispánicos. México: Instituto de Investigaciones Históricas, Universidad Nacional Autónoma de México.

Castillo Farreras, Víctor

1984 Estructura económica de la sociedad mexica según las fuentes documentales. Cultura Náhuatl, Monografías 13. México: Instituto de Investigaciones Históricas, Universidad Nacional Autónoma de México.

Charlton, Thomas H. y Deborah L. Nichols

1997a «The City State Concept: Development and Applications», en The Archaeology of City States: Cross-Cultural Approaches, Deborah L. Nichols y Thomas H. Charlton, eds., pp. 1-14. Washington D.C.: Smithsonian Institution Press.

1997b «Diachronic Studies of City-States: Permutations on a Theme -Central Mexico from 1700 B.C. to A.D. 1600», en The Archaeology of City States: Cross-Cultural Approaches, Deborah L. Nichols y Thomas H. Charlton, eds., pp. 169-207. Washington D.C.: Smithsonian Institution Press.

Claessen, Henri J. M.

1979 Antropología política. Estudio de las comunidades políticas (Una investigación panorámica). México: Instituto de Investigaciones Jurídicas e Instituto de Investigaciones Antropológicas, Universidad Nacional Autónoma de México.

ClaviJero, Francisco Javier

1991 Historia antigua de México [1781]. México: Editorial Porrúa.

CóDice Aubin

1980 Códice Aubin [Códice de 1576]. Manuscrito Azteca de la Biblioteca Real de Berlín. México: Editorial Innovación.

Codice Azcatitlan

1995 Codice Azcatitlan. París: Bibliothèque Nationale de France - Société des Américanistes.

CóDICE Boturini

1975 Códice Boturini o Tira de la peregrinacion. México: Secretaria de Educación Pública.

CóDICE RAMÍREZ

1985 Códice Ramírez. Manuscrito del siglo XVI intitulado, Relación del origen de los indios que habitan esta Nueva España, según sus historias, edición de Manuel Orozco y Berra. México: Editorial Innovación.

CóDice Xoloth

1980 Códice Xolotl, edición de Charles E. Dibble. México: Instituto de Investigaciones Históricas, Universidad Nacional Autónoma de México.

CORONA SÁNCHEZ, Eduardo

1973 Desarrollo de un señorío en el Acolhuacan prehispánico. Tesis de Maestría. Escuela Nacional de Antropología e Historia, México.

Crider, Destiny, Deborah L. Nichols, Hector NeFF y Michael D. Glascock

2007 «In the Aftermath of Teotihuacan: Epiclassic Pottery Production and Distribution in the Teotihuacan Valley, Mexico». Latin American Antiquity 18 (2): 123-143.

DAVIES, Nigel

1973 Los mexicas. Primeros pasos hacia el Imperio. México: Instituto de Investigaciones Históricas, Universidad Nacional Autónoma de México. 
1980 The Toltec Heritage. From the Fall of Tula to the Rise of Tenochtitlan. Norman: University of Oklahoma Press.

1987 The Aztec Empire. The Toltec Resurgence. Norman: University of Oklahoma Press.

DurÁn, Fray Diego

1995 Historia de las Indias de Nueva España e Islas de Tierra Firme, edición de Ángel María Garibay. México: Editorial Porrúa.

FLANNERY Kent V.

1972 «The Cultural Evolution of Civilizations». Annual Review of Ecology and Systematics 3: 399-426.

Franco, José Luis

1945 «Comentarios sobre la decoración negra en la cerámica Azteca II». Revista Mexicana de Estudios Antropológicos 7: 163-186.

FRIED, Morton H.

1960 «On the Evolution of Social Stratification and the State», en Culture in History. Essays in Honor of Paul Radin, Stanley Diamond, ed., pp. 713-731. Nueva York: Columbia University Press.

GAMIO, Manuel, Franz BoAs y Adolfo Best

1990 Álbum de colecciones arqueológicas [1921]. México: Instituto Nacional de Antropología e Historia.

GARcía CHÁvez, Raúl

2007 «El altepetl como formación sociopolítica de la Cuenca de México. Su origen y desarrollo durante el Posclásico Medio». Arqueoweb: Revista sobre Arqueología en Internet 8 (2).

GiBson, Charles

1981 Los aztecas bajo el dominio español. México: Editorial Siglo XXI.

GrIFFIN James B. y Antonieta EsPEJO

1947 «La alfarería correspondiente al último periodo de ocupación nahua del Valle de México». Memorias de la Academia Mexicana de la Historia 6: 131-147.

HIRTH, Kenneth G.

2012 «El altepetl y la estructura urbana en la Mesoamérica prehispánica», en El poder compartido. Ensayos sobre la arqueología de organizaciones políticas segmentarias y oligárquicas, Annick Daneels y Gerardo Gutiérrez, coords., pp. 69-99. México: Centro de Investigación y Estudios Superiores en Antropología Social.

Hodge, Mary G.

1984 Aztec City-States. Memoirs of the Museum of Anthropology 18. Ann Arbor: University of Michigan.

1997 «When is a City State?», en The Archaeology of City States, Deborah L. Nichols y Thomas H. Charlton, eds. pp. 169-207. Washington D.C.: Smithsonian Institution Press.

Hodge, Mary G. y Leah D. MinC

1990 «The Spatial Patterning of Aztec Ceramics: Implications for Prehispanic Exchange Systems in the Valley of Mexico». Journal of Field Archaeology 17 (4): 415-437.

Hodge, Mary G. y Michael E. Smith (eds.)

1994 Economies and Polities in the Aztec Realm. Albany: Institute for Mesoamerican Studies. 
JiMÉNEZ MoRENO, Wigberto

1961 «Diferentes principios del año entre diversos pueblos y sus consecuencias para la cronología prehispánica». El México Antiguo 9: 137-152.

KIRCHHOFF, Paul

1954-55 «Calendario tenochca, tlatelolca y otros». Revista Mexicana de Estudios Antropológicos 14: 257-267.

1968 «Mesoamerica: Its Geographic Limits, Ethnic Composition and Cultural Characteristics», en Heritage of Conquest, Sol Tax, ed., pp. 17-30. Nueva York: Cooper Square Publishers.

1985 «El imperio tolteca y su caída», en Mesoamérica y el Centro de México. Una antología, Jesús Monjarás-Ruiz, Rosa Brambila y Emma Pérez Rocha, recop., pp. 249272. México: Instituto Nacional de Antropología e Historia.

KIRChHOFF, Paul, Lina Ódena GüEmes y Luis ReYes GARCíA

1976 Historia Tolteca Chichimeca. México: Centro de Investigaciones Superiores del Instituto Nacional de Antropología e Historia.

MANZANILla, Linda

2001 «Gobierno corporativo en Teotihuacan: una revisión del concepto ‘palacio' aplicado a la gran urbe prehispánica». Anales de Antropología 35: 157-190.

MAPA QUINATZIN

1891 «Mapa Quinatzin. Cour Chichimeque et Histoire de Tezcuco», en Documents pour servir à l'histoire du Mexique, Vol. 1, Eugène Boban, ed., pp. 219-242, láminas 211212. Paris: Ernest Leroux

MAPA TLOTZIN

1885 Mémoires sur la peinture didactique et l'écriture figurative des anciens mexicains, edición de Joseph M. A. Aubin. París: Imprimerie Nationale.

MOHAR Betancourt, Luz María

1987 El tributo mexica en el siglo XVI. Análisis de dos fuentes pictográficas. México: CIESAS.

2004 Códice Mapa Quinatzin. Justicia y derechos humanos en el México Antiguo. México: CIESAS - Comisión Nacional de Derechos Humanos.

Moragas Segura, Natalia

2005 «Sobreviviendo al colapso: teotihuacanos y coyotlatelcos en Teotihuacan». Revista Española de Antropología Americana 35: 33-50.

Noguera, Eduardo

1935 «La cerámica de Tenayuca y las excavaciones estratigráficas», en Tenayuca: estudio arqueológico de la pirámide de este lugar, Eduardo Noguera, ed., pp. 141201. México: Talleres Gráficos del Museo Nacional de Arqueología, Historia y Etnografía.

O’NeILl, George C.

1962 Postclassic Ceramic Stratigraphy at Chalco in the Valley of Mexico. Tesis doctoral, Faculty of Political Science, Columbia University.

PARSONS, Jeffrey R.

1966 The Aztec Ceramic Sequence in the Teotihuacan Valley, Mexico. Tesis doctoral, Department of Anthropology, The University of Michigan. 
PÉRez Flores, José Luis

2013 «La mirada del poder y las permutaciones de la imagen de los chichimecas en textos del siglo XVI», en Poder y alteridad: perspectivas desde la antropología, la literatura y la historia, Ramón M. Pérez Martínez y Sergio A. González Varela, coords., pp. 193-220. Pontevedra: Editorial Academia del Hispanismo.

PÉREz Rocha, Emma

1982 La tierra y el hombre en la Villa de Tacuba durante la época colonial. México: Instituto Nacional de Antropología e Historia.

Pomar, Juan Bautista

1975 Relación de Texcoco (siglo XVI), edición facsimilar de la de 1891 con advertencia preliminar y notas de Joaquín García Icazbalceta. México: Biblioteca Enciclopédica del Estado de México.

REYes GARCÍA, Luis

1988 Cuautinchan del siglo XII al XVI. Formación y desarrollo histórico de un señorio prehispánico. México: CIESAS, FCE, Gobierno del Estado de Puebla.

SANDERS, William T., Jeffrey R. PARsons y Robert S. SANTLEY

1979 The Basin of Mexico: Ecological Processes in the Evolution of a Civilization. Nueva York: Academic Press.

Santamarina Novillo, Carlos

2015 «Salvajes y chichimecas: mitos de alteridad en las fuentes novohispanas». Revista Española de Antropología Americana 45 (1): 31-56.

SARMiento, Griselda

1992 Las primeras sociedades jerárquicas. México: Instituto Nacional de Antropología e Historia.

SCHROEDER, Susan

1994 Chimalpahin y los reinos de Chalco. México: El Colegio Mexiquense y Ayuntamiento Constitucional de Chalco.

SEJOURNÉ, Laurette

1970 Arqueología e historia del Valle de México: 1. Culhuacán. México: Instituto Nacional de Antropología e Historia.

SERVICE, Elman R.

1971 Cultural Evolutionism: Theory in Practice. Nueva York: Holt, Rinehart \& Winston.

1975 Origins of the State and Civilization. The Process of Cultural Evolution. Nueva York: W.W. Norton \& Company.

SMITH, Michael E.

1996 The Aztecs. Cambridge: Blackwell.

Smith, Michael E. y Mary G. Hodge

1994 «An introduction to Late Posclassic Economies and Polities», en Economies and Polities in the Aztec Realm, Mary G. Hodge y Michael E. Smith, eds., pp. 313-348. Albany: Institute for Mesoamerican Studies.

TORQuemadA, Fray Juan de

1975 Monarquía indiana, 7 vols. México: Instituto de Investigaciones Históricas, Universidad Nacional Autónoma de México.

VeYtia, Mariano

1979 Historia antigua de México. México: Editorial del Valle de México. 


\section{ZANTWIJK, Rudolf van}

1969 «La estructura gubernamental del Estado de Tlacupan (1430-1520)». Estudios de Cultura Náhuatl 8: 123-155.

1975 «El origen de la sociedad y del estado aztecas y la historicidad de las fuentes autóctonas, una introducción». Boletín de Estudios Latinoamericanos y del Caribe 18: 4-14.

1985 The Aztec Arrangement. The Social History of Pre-Spanish Mexico. Norman: University of Oklahoma Press. 